\title{
Orientaciones para el diseño de planes de formación del profesorado de las escuelas rurales de Catalunya
}

\author{
Roser Boix Tomàs* \\ Francesc Buscà Donet ${ }^{* *}$
}

\section{Resumen}

La escuela rural tiene una función clave para arraigar la comunidad escolar con el territorio, y al mismo tiempo para visibilizar y fortalecer su presencia en la población a la cual pertenece. Para ello, el profesorado de estas escuelas necesita de una formación específica que le proporcione los conocimientos, las habilidades y las actitudes que garanticen el desempeño de esta función. En este artículo se presentan unas orientaciones para diseñar esta formación específica fundamentada en los resultados obtenidos en el proyecto FOPROMAR-Erasmus plus (2017-1-ES01-KA201-038217). En concreto se parte de los datos obtenidos a partir del Cuestionario de Competencias y Saberes del Profesorado de la Escuela Rural administrado a maestros y maestras rurales catalanes. Los resultados apuntan que: 1) estos planes de formación específica deberían tener presente tanto competencias como saberes vinculados con el desempeño de la dimensión territorial; 2) que las competencias y saberes a seleccionar deberían basarse en la opinión del propio profesorado.

\section{Palabras clave}

Formación del profesorado, formación inicial, formación permanente, escuela rural, dimensión territorial.

\section{Recepció original: 21 d'abril de 2020}

Acceptació: 14 de juliol de 2020

Publicació: 16 de desembre de 2020

\section{Introducción'}

La educación en contextos rurales y por extensión, la escuela rural apenas ha sido contemplada como objeto a tener en cuenta en la formación del profesorado. Este hecho se puede constatar si se consultan la ORDEN ECI/3854/2007, de 27 de diciembre, por la que se establecen los requisitos para la verificación de los títulos universitarios oficiales que habiliten para el ejercicio de la profesión de Maestro en Educación Infantil, y en la Orden $\mathrm{ECl} / 3857 / 2007$, de 27 de diciembre, por la que se establecen los requisitos para la verificación de los títulos universitarios oficiales que habiliten para el ejercicio de la profesión de Maestro en Educación Primaria. Del mismo modo, la escuela rural tampoco ha sido tenida en cuenta de forma explícita en los correspondientes planes de estudio; en la formulación de las competencias profesionales básicas, generales y específicas y tampoco

$\left.{ }^{*}\right)$ Doctora por la Universidad de Barcelona y licenciada en Ciencias de la Educación por la Universidad de Barcelona. Su ámbito de estudio es la escuela rural y la educación en territorios rurales. Dirección electrónica: roser.boix@ub.edu

$\left(^{* *}\right)$ Doctor por la Universidad de Barcelona y licenciado en Educación Física por el INEFC-Universitat de Lleida. Su ámbito de estudio se centra en el desempeño de las competencias básicas a través de la educación física escolar. Miembro del GISEAFE (Grup d'investigació Social i Educativa de l'Activitat Física i I'Esport) (2017SGR1162) del INEFC-Universidad de Barcelona. Dirección electrónica: fbusca@ub.edu

(1) Este estudio forma parte del proyecto La Formación profesional y las competencias del maestro rural como dinamizador de la dimensión territorial en la escuela (2017-1-ES01-KA201-038217), subvencionado por la Unión Europea en el marco del programa Erasmus + 2017 (Acción KA201-Asociaciones estratégicas). 
en los contenidos y los resultados de aprendizaje de las diferentes materias que conforman la planificación de las enseñanzas.

Ante esta coyuntura, hace más de veinte años, las facultades de educación de las universidades catalanas crearon el Grupo Interuniversitario de Escuela Rural (GIER). Su objetivo principal era impulsar el estudio de la escuela rural desde los programas docentes de determinadas asignaturas de formación básica, obligatorias y optativas. El GIER ha sido el responsable de que, en la actualidad, estas facultades traten la escuela rural en los planes de estudio de los grados de maestro; y de que el alumnado pueda conocer esta tipología de escuela a partir de las prácticas externas, de optativas de escuela rural o bien, a través de asignaturas como, por ejemplo: sistema educativo y organización escolar, sociología, observación e innovación en el aula, entre otras.

A pesar de ello, es necesario reconsiderar la necesidad de la adquisición de competencias profesionales específicas para el trabajo en la práctica pedagógica del aula rural. Ésta se sitúa en el marco de una dimensión territorial que, por lógica, es muy distinta de la urbana. El dominio de competencias que le permitan al maestro en formación incorporar las características del contexto rural en su planificación curricular, y dar respuesta a la diversidad de edades que conviven en la clase multigrado, es fundamental para una educación de calidad y de justicia social en los territorios rurales. Un maestro que se forme solo para el trabajo pedagógico en una escuela ordinaria recibe una formación muy sesgada de la diversidad de centros escolares existentes en nuestro país. Y en ningún momento se puede pretender que esa formación también le sirva para instruir en un aula rural. No es un proceso de adaptación urbana lo que el maestro necesita para ejercer su profesión en la ruralidad, sino un conocimiento definido de la didáctica multigrado y la realidad de la educación en los territorios rurales. En definitiva, unas competencias específicas que le permitan ajustarse a las necesidades reales de la práctica pedagógica rural.

Sobre la base de esta problemática se planteó el diseño el proyecto de investigación La formación profesional y las competencias del maestro rural como dinamizador de la dimensión territorial de la escuela rural (FOPROMAR, Erasmus Plus, 2017-1-ESO1-KA201038217). Los objetivos generales de este proyecto eran los siguientes:

1. Identificar y analizar las competencias específicas del maestro rural vinculadas con la dimensión territorial de la escuela (entorno social y natural).

2. Identificar y analizar los saberes latentes en la escuela rural relacionados con la dimensión territorial de la escuela (entorno social y natural).

3. Elaborar una propuesta de plan de formación permanente y propuestas de formación inicial.

En el proyecto han participado seis socios: la Fundació del Món Rural (coordinadora del proyecto), la Universidad de Barcelona, la Universidad de Zaragoza, la Universidad de Lisboa, la Universidad de Burdeos y la Federación de Movimientos de Renovación Pedagógica de Cataluña. La duración del proyecto ha sido de dos años (2017-2019).

El principal propósito de este artículo es dar respuesta al tercer y último de estos objetivos para la Comunidad Autónoma de Cataluña. En concreto, pretende identificar 
aquellas competencias y saberes relacionados con la dimensión territorial que los agentes responsables de la formación específica del profesorado de la escuela rural podrían tener como referente a la hora de diseñar sus planes formativos.

En este trabajo dichas orientaciones se fundamentan en la opinión del propio profesorado de la escuela rural catalana. Es por este motivo que, en el marco del proyecto FOPROMAR, se estimó oportuno diseñar y validar un cuestionario de encuesta dirigido a este colectivo, y con la intención de:

a) Identificar qué competencias y saberes relacionados con la dimensión territorial, son los más destacados por el profesorado de la escuela rural en cuanto a su grado de relevancia.

b) Identificar qué competencias y saberes relacionados con la dimensión territorial han sido los que menor puntuación han obtenido con respecto a su grado de dominio.

\section{Las competencias y los saberes profesionales}

En el año 2000 el Consejo de Europa en Lisboa (Comisión Europea) propuso que la Unión Europea se convirtiera en la región más competitiva del mundo en términos de economía del conocimiento. Para ello se determinó que la formación de docentes debería integrar el concepto de «competencia» como eje central en el marco de la educación superior (aunque también en la primaria y la secundaria). En el contexto académico, la noción de competencia se considera una combinación de actitudes, aptitudes y capacidades, que con una formación adecuada permite al estudiante analizar una situación específica y actuar en consecuencia. (Cano, 2011).

Se parte de un principio basado en la integración y contextualización de estas actitudes, aptitudes y capacidades. No obstante, se trata de un término complejo que ha dado lugar a múltiples definiciones, que van desde la concepción de ser una característica subyacente en un individuo (Spencer y Spencer, 1993), pasando por un conjunto de comportamientos observables que llevan a un desempeño efectivo en un contexto específico (Pereda, Berrocal y López, 2002), hasta el buen desempeño en contextos diversos y auténticos basados en la integración y activación de conocimientos, normas, etc. (Universidad de Deusto, 2006).

Desde nuestro punto de vista, ser un profesional competente implica dominar todos los elementos, no sólo algunos de ellos. Así, sin perder este significado integrador, encontramos varias características que definen el significado global del término.

En primer lugar, se trata de un «saber» y de un «saber hacer». La competencia se manifiesta y adquiere todo su significado en la acción. Y para ello es imprescindible poder llevarla a cabo en las distintas situaciones que puedan surgir, es decir, que sea susceptible a adaptarse a los distintos contextos, además de multifuncional y transferible. Otra característica es su naturaleza integradora, como decíamos, en el sentido de que engloba de forma natural, conocimientos, procedimientos y actitudes. Para Perrenoud (2004), las competencias no son en sí mismas conocimientos, capacidades o actitudes, a pesar que movilicen, integren u orquesten diferentes recursos cognitivos. En este proceso, también se activan patrones de percepción, de evaluación, de anticipación y de toma de decisiones que conducen a una cierta capacidad de acción. 
La Unión Europea ha estado trabajando para el desarrollo de una política de calidad en materia de formación del profesorado con la intención de describir las principales líneas de formación y las aptitudes y competencias necesarias para los docentes (OliverTrobat, Forteza y Urbina, 2015).

En el contexto de esta investigación, sobre la base de estas recomendaciones y referentes extraídos de Perrenaud (2004) y del informe Eurydice (2012) se ha elaborado una clasificación en torno a tres grandes bloques o ámbitos de competencias y docentes que los maestros y maestras que trabajen en territorios rurales deberían ser capaces de desempeñar. Estos ámbitos son los siguientes: pedagógico, metodológico y relacional.

Tal y como se expondrá en el siguiente apartado, cada uno de estos ámbitos incluyen otras más específicas, cuyos criterios de diseño y organización fueron elaborados y consensuados por el equipo de investigación de FOPROMAR.

En lo que respecta a los saberes docentes relacionados con el contexto rural territorio y que el profesorado de escuela rural debería dominar para el desempeño de su labor docente, se ha partido de las definiciones de patrimonio material e inmaterial de la UNESCO. El patrimonio material se considera el cultural y el natural; mientras que el patrimonio inmaterial contempla las tradiciones y expresiones orales, las artes escénicas, las prácticas sociales, rituales y eventos festivos, conocimientos y prácticas sobre la naturaleza y el universo, y los conocimientos y costumbres relacionados con la artesanía tradicional (Convención para la salvaguardia del patrimonio cultural inmaterial, 2003).

\section{Método}

Este estudio de naturaleza descriptiva se corresponde con un diseño de investigación no experimental basado en la administración de un cuestionario de encuesta (McMillan y Schumacher, 2011).

\section{Muestra}

La muestra de este estudio se compone de 276 docentes de las escuelas rurales catalanas. Según los datos que dispone el Departament d'Educació de la Generalitat de Catalunya, la población actual de maestros y maestras rurales es de 2766. Por tanto, puede concluirse que con un margen de error del $5 \%$, la muestra seleccionada es representativa en más de un $90 \%$.

\section{Instrumento}

El Cuestionario Competencias y Saberes del Profesorado de la Escuela Rural (CCS-PER) se compone de tres partes: datos personales y de contexto; competencias profesionales y saberes.

La primera parte se compone de variables que permiten obtener información en cuanto a la edad, género y años de experiencia en la enseñanza y la escuela rural de los maestros y maestras, así como de la población y la escuela rural en la que desempeñan su labor docente en Cataluña.

La segunda parte se configura en torno a una selección de 30 competencias relacionadas con el desempeño de la dimensión territorial en la escuela rural (tabla 1). 
Estas competencias se agrupan en los ámbitos competenciales referidos anteriormente, y se concretan del siguiente modo:

- Ámbito pedagógico: agrupa 6 competencias clave (ítems del 1 al 6) en las que el profesorado desempeña su capacidad para adecuar su proyecto pedagógico personal a las características y necesidades tanto del entorno como de implicar y colaborar con el resto de docentes para desarrollar proyectos compartidos vinculados al territorio.

- Ámbito metodológico: contiene 17 competencias instrumentales (ítems del 7 al 23) en las que los y las docentes muestran su capacidad para diseñar, gestionar y evaluar proyectos educativos, propuestas didácticas y recursos en las que se tenga en cuenta las problemáticas y retos del territorio y el alumnado.

- Ámbito relación escuela-territorio: agrupa 7 competencias de carácter sistémico (ítems del 24 al 30) en la que el profesorado muestra su capacidad para fomentar implicar y fomentar la participación en los proyectos educativos y las propuestas didácticas de los miembros de la comunidad escolar y los agentes sociales y educativos del territorio.

\section{Tabla 1. Relación de competencias profesionales del cuestionario CCS-PER}

\begin{tabular}{|c|c|c|}
\hline Ámbito & Ítem & Competencia \\
\hline \multirow[t]{6}{*}{ Pedagógico } & 1 & Considerar las necesidades de su territorio rural en la planificación docente. \\
\hline & 2 & $\begin{array}{l}\text { Incluir en la planificación docente las características culturales de su territo- } \\
\text { rio rural. }\end{array}$ \\
\hline & 3 & $\begin{array}{l}\text { Identificar las necesidades formativas del profesorado del centro en relación } \\
\text { con su territorio rural. }\end{array}$ \\
\hline & 4 & $\begin{array}{l}\text { Posibilitar acciones de desarrollo profesional para el profesorado del centro } \\
\text { en relación con las necesidades formativas detectadas. }\end{array}$ \\
\hline & 5 & Implicar al equipo docente en los proyectos vinculados a su territorio rural. \\
\hline & 6 & $\begin{array}{l}\text { Incluir las necesidades de su territorio rural en el diseño de los proyectos de } \\
\text { innovación. }\end{array}$ \\
\hline \multirow[t]{12}{*}{ Metodológico } & 7 & $\begin{array}{l}\text { Sensibilizar al alumnado sobre las necesidades relacionados con el desarro- } \\
\text { llo sostenible de su territorio rural. }\end{array}$ \\
\hline & 8 & Desarrollar el conocimiento de la diversidad cultural de su territorio rural. \\
\hline & 9 & Desarrollar una actitud crítica ante los valores de su territorio rural. \\
\hline & 10 & $\begin{array}{l}\text { Tener en cuenta los conocimientos previos relacionados con la cultura de su } \\
\text { territorio rural. }\end{array}$ \\
\hline & 11 & $\begin{array}{l}\text { Establecer relaciones entre las experiencias previas vinculadas a su territorio } \\
\text { rural y los nuevos aprendizajes. }\end{array}$ \\
\hline & 12 & Relacionar los contenidos con la realidad territorial inmediata. \\
\hline & 13 & $\begin{array}{l}\text { Proponer situaciones de investigación relacionadas con el entorno rural pró- } \\
\text { ximo. }\end{array}$ \\
\hline & 14 & Desarrollar actividades para el conocimiento de la cultura local. \\
\hline & 15 & Realizar actividades complementarias que partan del territorio rural. \\
\hline & 16 & $\begin{array}{l}\text { Considerar las características de su territorio rural en la organización de los } \\
\text { espacios y tiempos escolares. }\end{array}$ \\
\hline & 17 & $\begin{array}{l}\text { Utilizar los recursos que ofrece su territorio rural (naturales, materiales, socia- } \\
\text { les y culturales). }\end{array}$ \\
\hline & 18 & Implicar en el centro a agentes de la comunidad local. \\
\hline
\end{tabular}




\begin{tabular}{|c|c|c|}
\hline & 19 & $\begin{array}{l}\text { Elaborar materiales curriculares con participación de agentes de su territorio } \\
\text { rural. }\end{array}$ \\
\hline & 20 & Incorporar el uso de TIC para el conocimiento de territorio rural próximo. \\
\hline & 21 & $\begin{array}{l}\text { Utilizar las TIC como elemento de comunicación con los agentes de su terri- } \\
\text { torio rural. }\end{array}$ \\
\hline & 22 & $\begin{array}{l}\text { Considerar los saberes sobre la cultura local en la evaluación de los aprendi- } \\
\text { zajes. }\end{array}$ \\
\hline & 23 & $\begin{array}{l}\text { Considerar los contenidos relacionados con el desarrollo sostenible de su te- } \\
\text { rritorio rural en la evaluación de los aprendizajes. }\end{array}$ \\
\hline \multirow{7}{*}{$\begin{array}{l}\text { Relación } \\
\text { escuela- } \\
\text { comunidad }\end{array}$} & 24 & $\begin{array}{l}\text { Implicar a la comunidad local en el diseño, desarrollo y evaluación del pro- } \\
\text { yecto educativo de centro incorporando sus expectativas. }\end{array}$ \\
\hline & 25 & $\begin{array}{l}\text { Incluir en los objetivos de la escuela el servicio al desarrollo cultural de su te- } \\
\text { rritorio rural. }\end{array}$ \\
\hline & 26 & Comprometerse con iniciativas socio-culturales de la comunidad. \\
\hline & 27 & $\begin{array}{l}\text { Establecer procesos de participación escuela-familias-comunidad y vice- } \\
\text { versa. }\end{array}$ \\
\hline & 28 & Implicar a la familia en actividades extraescolares. \\
\hline & 29 & Implicar a la comunidad local en actividades extraescolares. \\
\hline & 30 & Utilizar la escuela como espacio cultural de la comunidad local. \\
\hline
\end{tabular}

Fuente: Elaboración propia a partir de la validación del cuestionario

La tercera y última parte del cuestionario se compone de una selección de 27 saberes relacionados con el patrimonio económico, social y cultural propio de los territorios rurales (Tabla 2).

Estos saberes se agrupan en los ámbitos que se detallan a continuación:

- Contenidos relacionados con la literatura, tradiciones y artes locales (ítems del 1 al 11).

- Contenidos relacionados con la organización social e institucional del territorio rural (ítems del 12 al 18).

- Contenidos representativos del entorno y el patrimonio natural del territorio (ítems del 19 al 22).

- Conocimientos destacados del patrimonio cultural e industrial del territorio (ítems del 23 al 27).

Tabla 2. Relación de saberes del cuestionario CCS-PER

\begin{tabular}{lcll}
\multicolumn{1}{c}{ Ámbito } & Ítem & & Saber \\
\hline \multirow{2}{*}{ Literatura, tradiciones y artes locales } & 1 & Literatura popular & \\
2 & Proverbios \\
3 & Mitos \\
4 & Rituales \\
5 & Creencias \\
6 & Juegos \\
7 & Gastronomía \\
8 & Música \\
9 & Danzas \\
10 & Teatro \\
11 & Artesanía \\
\hline
\end{tabular}




\begin{tabular}{lll}
\hline \multirow{2}{*}{ Organización social } & 12 & Prácticas sociales \\
& 13 & Fiestas \\
& 14 & Organización local \\
15 & Administraciones \\
& 16 & Instituciones culturales \\
& 17 & Protección medio ambiente \\
& 18 & Conocimiento de otras instituciones locales \\
\hline \multirow{2}{*}{ Naturaleza y entorno } & 19 & Ecología \\
& 20 & Flora y fauna \\
& 21 & Patrimonio natural \\
& 22 & Patrimonio agrícola-forestal \\
\hline \multirow{2}{*}{ Patrimonio mueble e inmueble } & 23 & Mueble artístico \\
& 24 & Mueble no artístico \\
25 & Inmueble \\
26 & Industrial \\
27 & Subacuático-subterráneo \\
\hline
\end{tabular}

Fuente: Elaboración propia a partir de la validación del cuestionario

Para la selección de las competencias y saberes que componen el CCS-PER se procedió del siguiente modo. En primer lugar, se llevó a cabo un proceso previo de documentación. Los referentes teóricos consultados (Comisión Europea, 2004; European Comission, 2005, 2007; González y Wagenaar, 2010; Perrenaud, 2007; UNESCO, 2014) permitieron confeccionar un listado provisional de competencias y saberes. A continuación, este listado se sometió a consideración de investigadores y profesorado con experiencia en la escuela rural con la intención de validar el contenido de cada uno de los ítems del cuestionario. Finalmente, el cuestionario fue sometido a un pilotaje previo, con la intención de constatar su fiabilidad. Para ello se aplicó el índice alfa de Cronbach, cuyo resultado permitió constatar una alta consistencia interna $(a=.97)$.

\section{Procedimiento}

El cuestionario se envía a los maestros y maestras de las escuelas rurales de Catalunya en formato electrónico, a través de la aplicación google forms.

Teniendo en cuenta las dificultades para acceder in situ a todo el profesorado de las escuelas rurales (generalistas e itinerantes), se empleó un método no probabilístico por bola de nieve. Para ello se utilizó la base de datos del Secretariat d'Escola Rural de Catalunya con la intención de enviar el cuestionario a la dirección personal e institucional de correo electrónico de todos los directores de las escuelas censadas en el Departament d'Educació. Además de responder el cuestionario, los miembros del equipo directivo recibieron el encargo de remitirlo al profesorado de su escuela para que éstos pudieran reenviarlo cumplimentado a los responsables del estudio.

La persona encuestada debía puntuar por medio de dos escalas de Likert el grado de relevancia y de dominio de las competencias y saberes incluidos en el cuestionario. En ambos casos, las puntuaciones se correspondían con los siguientes valores: 1 (nada); 2 (reducido); 3 (mediano) y 4 (elevado). 
La recepción de cuestionarios se mantuvo activa prácticamente durante un año natural, procurando evitar los finales y los inicios de curso y, por tanto, los periodos en que el profesorado suele tener mayores dificultades para responder el cuestionario.

\section{Análisis de los datos}

En primer lugar, para comprobar en qué grado las puntuaciones obtenidas para cada ítem son representativas del ámbito de competencias o de saberes al cual pertenecen se realiza el análisis de consistencia interna (alfa de Cronbach). Para establecer el tipo de relación existente entre las puntuaciones otorgadas, tanto al valorar el grado de dominio, como de relevancia de las competencias y los saberes indicados en el cuestionario CCS$P E R$, se calcula el Índice de correlación de Pearson. Con ello, se espera determinar si el hecho que una competencia o saber sea valorado con una determinada puntuación en cuanto a su dominio, también incide -y de qué modo- en la puntuación otorgada en cuanto a su relevancia.

Para identificar qué competencias y saberes relacionados con la dimensión territorial han sido los que han obtenido mayores y menores puntuaciones medias, en función del grado de relevancia y dominio, se calcularon los estadísticos descriptivos básicos: media, mediana, desviación estándar y varianza.

Con estos resultados obtenidos de la opinión del profesorado de la escuela rural, se espera disponer de evidencias objetivas que permitan destacar qué competencias y saberes podrían constituirse como una referente a considerar a la hora de proponer un hipotético plan específico de formación del profesorado.

\section{Resultados}

La presentación de resultados se realiza en función de los objetivos planteados en este estudio. Se parte del supuesto de que cualquier plan específico de formación inicial o continuada del profesorado de la escuela rural debería de contemplar el desempeño de unas competencias profesionales y saberes específicos, y que estos pueden seleccionarse teniendo en cuenta la opinión de los maestros y maestras que están ejerciendo su labor docente en el territorio rural. Por otra parte, a partir de los resultados obtenidos tras la administración del cuestionario CCS-PER, se entiende también que los mejores indicadores a considerar para esta selección serán, por un lado, las competencias y saberes mejor puntuados en cuanto a su relevancia, $y$, por otro, las competencias y saberes que menores puntuaciones han obtenido en cuanto a su dominio.

\section{¿Qué tipo de relación existe entre las puntuaciones obtenidas en cuanto a la rele- vancia y el dominio de estas competencias y saberes?}

Antes de analizar las puntuaciones otorgadas por los encuestados a la hora de valorar las competencias y saberes destacados en el cuestionario, es necesario constatar qué tipo de relación existe entre las puntuaciones computadas en cuanto al grado de relevancia y dominio. De este modo, se podrá estimar si el hecho que una competencia o saber haya sido valorado como relevante con una determinada puntuación también influye en la puntuación otorgada por el profesorado a la hora de evaluar su grado de desempeño. 
Tras aplicar el coeficiente de correlación de Pearson entre las puntuaciones otorgadas en cada una de las categorías de competencias y saberes contemplados en este estudio, los resultados señalan que existe:

- Un alto grado de significación entre las medias de las puntaciones obtenidas al valorar la relevancia y el dominio de las competencias pedagógicas $(r=0.966 ; p<$ $0.001 ;$ gl.5).

- Un grado de significación fuerte entre las puntuaciones registradas entre las competencias metodológicas $(\mathrm{r}=0,918 ;<0.001 ; \mathrm{gl} .15)$; los saberes relacionados con la organización social $(r=0,888 ; p<0.01 ; g l .6)$ y los saberes vinculados con la naturaleza y el entorno rural $(r=0,954 ; p<0.02 ; g l .3)$

- Un grado significativo entre las puntuaciones obtenidas en los saberes relacionados con el patrimonio mueble e inmueble $(r=0,841 ; p<0.05 ; \mathrm{gl} .4)$.

- La ausencia de significación entre las puntuaciones obtenidas en los saberes relacionados con la lengua, tradiciones y artes locales ( $r=0,475 ; p>0.05 ; g l .10)$.

Estos resultados permiten constatar que, cuando se otorga una puntuación alta o baja para determinar la relevancia de la mayoría de las competencias y los saberes destacados, se observa la misma tendencia a la hora de valorar el grado de domino. Este hecho es muy relevante para deducir qué competencias y saberes podrían seleccionarse a la hora de diseñar propuestas formativas específicas. Por este motivo, a continuación, se describen cuáles han sido las que han recibido las puntuaciones más destacadas en cuanto su relevancia y las menos puntuadas en cuanto a su grado de dominio.

\section{¿Qué competencias y saberes están mejor valoradas en cuanto a su relevancia?}

En líneas generales, los resultados muestran que el profesorado encuestado califica como elevada la importancia de las competencias relacionadas con la dimensión territorial (tabla 3). En este sentido, se observa que las puntuaciones medias oscilan entre un rango que va desde los 3.25 a los 3.76 puntos.

Por ámbitos competenciales, la misma tabla de resultados permite observar que, en lo que respecta al ámbito pedagógico, todos los resultados obtenidos muestran un índice elevado de consistencia interna $(a=.85)$. Por otra parte, la competencia más valorada es la competencia núm. 2 (Incluir en la planificación docente las características culturales de su territorio rural; $M=3.61$ ) En este caso, tanto la desviación típica como la varianza confirman el consenso existente con respecto a esta relevancia ( $D E=.55 ; \sigma=.30$ ).

En lo que respecta al ámbito metodológico, el índice de consistencia interna también muestra que las respuestas referidas a su pertinencia son muy consistentes $(a=.95)$. Asimismo, la competencia con una mayor puntuación media ha sido la 17 [Utilizar los recursos que ofrece su territorio rural (naturales, materiales, sociales y culturales; $\mathrm{M}=3.76$ ]. Al igual que en el caso anterior, la desviación típica y la varianza de las puntuaciones otorgadas por los encuestados revelan un elevado consenso en cuanto a la importancia de la competencia más valorada ( $\mathrm{DE}=.45 ; \sigma=.21$ ).

Finalmente, en cuanto al ámbito relación escuela-comunidad, destacar también que todas las puntuaciones que valoran las competencias de este ámbito sistémico son pertinentes, y muestran un índice elevado de consistencia interna $(a=.87)$. En este caso, la 
competencia mejor puntuada ha sido la n²7 (Establecer procesos de participación escuelafamilias-comunidad y viceversa; $M=3.61$ ). Siguiendo con la misma tendencia que en el resto de ámbitos, tanto la desviación típica como la varianza confirmarían un alto grado de consenso al respecto ( $\mathrm{DE}=.55 ; \sigma=.30)$.

En el caso de los saberes, los resultados indican que los encuestados, en general, valoran su relevancia con unas puntaciones que se sitúan en nivel medio-elevado (tabla 4), observándose un rango que oscila entre los 2.98 y los 3.63 puntos.

Por ámbitos de saberes, en primer lugar, conviene destacar que en el caso de los saberes relacionados con las lenguas, tradiciones y artes del territorio se observa un índice elevado de consistencia interna $(a=.96)$. De todos ellos, el saber $n^{\circ} 1$ ha sido el que ha obtenido una mayor puntuación (Literatura popular; $\mathrm{M}=3.42$ ). Esta opinión está plenamente corroborada al comprobar que tanto su desviación típica $(D E=.65)$ como, sobre todo, su varianza $(\sigma=.45)$, son de las que menos divergencia presentan.

En cuanto al ámbito de saberes relacionados con la organización social, también se observa un elevado índice de consistencia interna $(a=.93)$. En este caso, el saber núm. 13 (Fiestas populares; $\mathrm{M}=3.55$ ) es el mejor puntuado. A juzgar por la desviación típica $(\mathrm{DE}=.59)$ y por la varianza $(\sigma=.34)$ también podría decirse que esta opinión cuenta con un elevado grado de consenso.

El ámbito de saberes relacionados con la naturaleza y el entorno del territorio también muestra un elevado índice de coherencia interna $a=.93$ ). Aquí el saber núm. 21 (Patrimonio natural; $M=3.47$ ) es el puntuado como el más relevante y también es el que genera un mayor índice de consenso, a juzgar por los índices registrados en lo que respecta a su deviación $(\mathrm{DE}=.56)$ y varianza $(\sigma=.31)$.

Finalmente, en lo que se refiere a los saberes vinculados con el patrimonio mueble $e$ inmueble del territorio cabe destacar que su consistencia interna también es significativa $(a=.90)$, y que el saber con mayor puntuación ha sido el núm. 25 (Patrimonio inmueble; $M=3.47)$. Al igual que en todos los casos anteriores, la desviación típica ( $D E=.65)$ y la varianza $(\sigma=.42)$ confirmarían el consenso existente con respecto a esta valoración.

\section{Tabla 3. Descriptores del grado de dominio y} relevancia de las competencias profesionales

\begin{tabular}{|c|c|c|c|c|c|c|c|c|c|c|}
\hline \multirow[b]{2}{*}{ Ámbito } & \multirow[b]{2}{*}{$C$} & \multicolumn{4}{|c|}{ Dominio } & \multicolumn{4}{|c|}{ Relevancia } & \multirow{2}{*}{$\begin{array}{c}M_{\text {dom }} \\
M_{\text {rel }}\end{array}$} \\
\hline & & $M$ & med & $D E$ & $\sigma$ & $M$ & med & $D E$ & $\sigma$ & \\
\hline \multirow[t]{6}{*}{ Pedagógico } & 1 & 2.95 & 3 & .69 & .48 & 3.53 & 4 & .57 & .33 & -.58 \\
\hline & 2 & 3.15 & 3 & .67 & .45 & 3.61 & 4 & .55 & .30 & -.46 \\
\hline & 3 & 2.71 & 3 & .84 & .71 & 3.43 & 4 & .69 & .47 & -.72 \\
\hline & 4 & 2.68 & 3 & .84 & .70 & 3.41 & 3 & .66 & .43 & -.73 \\
\hline & 5 & 3.03 & 3 & .77 & .59 & 3.59 & 4 & .59 & .35 & -.56 \\
\hline & 6 & 2.84 & 3 & .86 & .75 & 3.53 & 4 & .61 & .37 & -.69 \\
\hline \multirow[t]{8}{*}{ Metodológico } & 7 & 3.05 & 3 & .78 & .60 & 3.67 & 4 & .51 & .26 & -.62 \\
\hline & 8 & 3.11 & 3 & .73 & .53 & 3.59 & 4 & .53 & .29 & -.48 \\
\hline & 9 & 3.05 & 3 & .76 & .58 & 3.65 & 4 & .54 & .29 & -.60 \\
\hline & 10 & 3.14 & 3 & .71 & .51 & 3.62 & 4 & .54 & .29 & -.48 \\
\hline & 11 & 3.13 & 3 & .69 & .48 & 3.61 & 4 & .54 & .29 & -.48 \\
\hline & 12 & 3.17 & 3 & .70 & .49 & 3.64 & 4 & .52 & .27 & -.47 \\
\hline & 13 & 2.95 & 3 & .86 & .75 & 3.60 & 4 & .57 & .32 & -.65 \\
\hline & 14 & 3.17 & 3 & .72 & .52 & 3.66 & 4 & .55 & .30 & -.49 \\
\hline
\end{tabular}




\begin{tabular}{|c|c|c|c|c|c|c|c|c|c|c|}
\hline & 15 & 3.00 & 3 & .79 & .63 & 3.46 & 4 & .63 & .40 & -.46 \\
\hline & 16 & 3.03 & 3 & .80 & .65 & 3.54 & 4 & .62 & .39 & -.51 \\
\hline & 17 & $3.29 *$ & 3 & .70 & .49 & $3.76^{*}$ & 4 & .45 & .21 & -.47 \\
\hline & 18 & 3.11 & 3 & .77 & .59 & 3.66 & 4 & .53 & .29 & -.55 \\
\hline & 19 & $2.49 * *$ & 2 & .92 & .85 & 3.29 & 3 & .74 & .54 & -.80 \\
\hline & 20 & 2.81 & 3 & .84 & .70 & 3.37 & 3 & .67 & .45 & -.56 \\
\hline & 21 & 2.77 & 3 & .88 & .78 & 3.37 & 3 & .67 & .45 & -.60 \\
\hline & 22 & 2.71 & 3 & .82 & .68 & $3.25 * *$ & 3 & .74 & .55 & -.54 \\
\hline & 23 & 2.71 & 3 & .82 & .67 & 3.32 & 3 & .69 & .48 & -.61 \\
\hline \multirow{7}{*}{$\begin{array}{l}\text { Relación escuela- } \\
\text { comunidad }\end{array}$} & 24 & 2.66 & 3 & .91 & .82 & 3.34 & 3 & .71 & .51 & -.68 \\
\hline & 25 & 2.61 & 3 & .87 & .76 & 3.31 & 3 & .69 & .48 & -.70 \\
\hline & 26 & 2.96 & 3 & .80 & .64 & 3.47 & 4 & .62 & .38 & -.51 \\
\hline & 27 & 3.12 & 3 & .77 & .59 & 3.61 & 4 & .55 & .30 & -.49 \\
\hline & 28 & 2.96 & 3 & .82 & .68 & 3.36 & 3 & .69 & .48 & -.40 \\
\hline & 29 & 2.71 & 3 & .85 & .72 & 3.24 & 3 & .72 & .52 & -.53 \\
\hline & 30 & 2.77 & 3 & .89 & .80 & 3.29 & 3 & .75 & .56 & -.52 \\
\hline
\end{tabular}

*Puntuación media más elevada; **puntuación media más baja

\section{¿Qué competencias y saberes son menos valoradas por su grado de dominio?}

Si bien es cierto que, para construir una propuesta formativa específica para el profesorado de la escuela rural, es importante conocer qué competencias y saberes son valorados como relevantes, estos planes tampoco pueden dejar de lado aquellas competencias y saberes que, desde el punto de vista de los propios encuestados, son los que menos domina. Sobre todo, si se tiene en cuenta que, en todos los casos, las puntuaciones otorgadas por el profesorado al valorar el dominio de estas competencias y saberes han sido ostensiblemente menores que al valorar su relevancia.

En el caso de las competencias, en primer lugar, se destaca que la tendencia general es que las valoraciones oscilen en un nivel reducido o bajo y mediano. Como puede observarse en la tabla 3 , las puntuaciones en cuanto a su dominio oscilan en un rango que va desde los 2.49 y los 3.29 puntos.

La misma tabla también muestra que, en lo que respecta al ámbito pedagógico, la competencia que menos puntuación ha recibido ha sido la núm. 4 (Posibilitar acciones de desarrollo profesional para el profesorado del centro en relación con las necesidades formativas detectadas; $M=2.68$ ). No obstante, si bien el índice de consistencia interna para este ámbito también es significativo ( $\mathrm{a}=.88$ ), la elevada desviación típica $(\mathrm{DE}=.84)$ y varianza $(\sigma=.70)$ inducen a pensar que existe una cierta dispersión a la hora de valorar esta competencia como la que menos dominan los encuestados.

En el caso de las competencias del ámbito metodológico, la que obtiene una menor puntuación ha sido la competencia núm. 19 (Elaborar materiales curriculares con participación de agentes de su territorio rural; $\mathrm{M}=2.49$ ). Tal y como sucede con el ámbito anterior, a pesar de la gran consistencia interna existente entre las respuestas obtenidas para este ámbito $(\mathrm{a}=.95)$, los elevados índices de desviación $(\mathrm{DE}=.92)$ y varianza $(\sigma=.85)$ muestran también la presencia de una gran dispersión.

Por último, al observar las puntuaciones obtenidas para las competencias del ámbito relación escuela-comunidad, se constata que la competencia con una menor puntuación se corresponde con la núm. 25 (Incluir en los objetivos de la escuela el servicio al desarrollo cultural de su territorio rural; $\mathrm{M}=2.61$ ). Siguiendo la misma tendencia que en los otros ámbitos competenciales, a pesar del elevado índice de consistencia interna $(\alpha=.90)$, también 
se observa una gran dispersión con respecto a las puntuaciones otorgadas a esta competencia $(\mathrm{DE}=.87 ; \sigma=.76)$.

En lo que respecta a los saberes que han obtenido menores puntuaciones por parte de los encuestados, en primer lugar, se debe destacar que las valoraciones se suelen situar en un nivel bajo o reducido. En concreto, la tabla 4 permite observar que las puntuaciones medias oscilan en un rango que va de los 3.29 a los 2.24 puntos.

En el caso del ámbito Lenguas, tradiciones y artes, el saber $n^{\circ} 2$ es el que presenta una menor puntuación (Proverbios populares; $\mathrm{M}=2.42$ ). Sin embargo, siguiendo la misma tendencia observada en las competencias con menor valoración, a pesar de la consistencia interna de las puntuaciones obtenidas en este ámbito $(\alpha=.95)$, tanto la desviación típica $(\mathrm{DE}=.90)$ como la varianza $(\sigma=.81)$ indican una gran dispersión a la hora de puntuar este saber en concreto.

Algo parecido sucede en el ámbito de saberes relacionados con la organización social del territorio. Aquí también se observa un elevado índice de consistencia interna ( $a=.93)$, siendo el saber núm. 18 el menos valorado por los encuestados en cuanto a su grado de dominio (Conocimiento de otras instituciones locales; $M=2.65$ ). Sin embargo, la desviación $(\mathrm{DE}=.90)$ de esta media de puntuaciones y la varianza $(\sigma=.80)$, confirman también una gran dispersión a la hora de otorgar estas valoraciones.

En lo que respecta al ámbito de saberes relacionados con la naturaleza y el entorno del territorio, el saber con una menor puntación ha sido el núm. 19 (Ecología, $M=2.71$ ). En este caso, la consistencia interna vuelve a ser elevada $(\alpha=.91)$ mientras que la desviación típica $(\mathrm{DE}=.88)$ y la varianza $(\sigma=.77)$ de las puntuaciones otorgadas a este saber, también muestran un elevado grado de dispersión.

Finalmente, al observar los saberes relacionados con el patrimonio mueble e inmueble, se destaca que el saber núm. 24 es el que recibe una menor puntuación media (Mueble no artístico; $M=2.24)$. Aquí también se constata un elevado grado de consistencia interna $(\mathrm{a}=.89)$ a la hora de valorar las puntuaciones medias que han recibido los saberes de este ámbito, al tiempo que una elevada dispersión en lo que respecta a las puntuaciones recibidas con respecto a este saber específico ( $D E=.90 ; \sigma=.80)$.

\section{Tabla 4. Descriptores correspondientes al grado de dominio y relevancia de los saberes}

\begin{tabular}{|c|c|c|c|c|c|c|c|c|c|c|}
\hline \multirow[b]{2}{*}{ Ámbito } & \multirow[b]{2}{*}{$S$} & \multicolumn{4}{|c|}{ Dominio } & \multicolumn{4}{|c|}{ Relevancia } & \multirow{2}{*}{$\begin{array}{c}M_{d o m}- \\
M_{r e l}\end{array}$} \\
\hline & & $M$ & med & $E T$ & Var & $M$ & med & $D E$ & $\sigma$ & \\
\hline \multirow{11}{*}{$\begin{array}{l}\text { Lenguas, tradiciones } \\
\text { y artes }\end{array}$} & 1 & 2.62 & 3 & .84 & .70 & 3.42 & 3 & .65 & .42 & -.80 \\
\hline & 2 & 2.42 & 2 & .90 & .81 & 3.27 & 3 & .72 & .52 & -.85 \\
\hline & 3 & 2.64 & 3 & .90 & .81 & 3.41 & 4 & .66 & .44 & -.77 \\
\hline & 4 & 2.64 & 3 & .89 & .79 & 3.28 & 3 & .75 & .56 & -.64 \\
\hline & 5 & 2.57 & 3 & .88 & .78 & 3.22 & 3 & .74 & .54 & -.65 \\
\hline & 6 & 2.71 & 3 & .87 & .76 & 3.41 & 3 & .62 & .38 & -.70 \\
\hline & 7 & 2.92 & 3 & .83 & .70 & 3.34 & 3 & .65 & .43 & -.42 \\
\hline & 8 & 2.71 & 3 & .91 & .83 & 3.39 & 3 & .64 & .41 & -.68 \\
\hline & 9 & 2.67 & 3 & .95 & .90 & 3.38 & 3 & .65 & .42 & -.71 \\
\hline & 10 & 2.53 & 3 & .97 & .95 & 3.23 & 3 & .77 & .59 & -.70 \\
\hline & 11 & 2.62 & 3 & .90 & .81 & 3.34 & 3 & .69 & .48 & -.72 \\
\hline Organización social & 12 & 2.78 & 3 & .83 & .68 & 3.33 & 3 & .70 & .48 & -.55 \\
\hline
\end{tabular}




\begin{tabular}{|c|c|c|c|c|c|c|c|c|c|c|}
\hline & 13 & 3.29* & 3 & .74 & .54 & 3.55 & 4 & .59 & .34 & -.26 \\
\hline & 14 & 2.97 & 3 & .78 & .61 & 3.38 & 3 & .69 & .48 & -.41 \\
\hline & 15 & 3.06 & 3 & .83 & .68 & 3.42 & 4 & .71 & .51 & -.36 \\
\hline & 16 & 3.02 & 3 & .81 & .65 & 3.45 & 4 & .67 & .45 & -.43 \\
\hline & 17 & 2.84 & 3 & .89 & .78 & 3.45 & 4 & .67 & .45 & -.61 \\
\hline & 18 & 2.65 & 3 & .90 & .80 & 3.23 & 3 & .76 & .58 & -.58 \\
\hline \multirow[t]{4}{*}{ Naturaleza y entorno } & 19 & 2.71 & 3 & .88 & .77 & 3.44 & 4 & .68 & .47 & -.73 \\
\hline & 20 & 2.87 & 3 & .85 & .72 & 3.58 & 4 & .62 & .38 & -.71 \\
\hline & 21 & 2.99 & 3 & .81 & .66 & $3.63^{*}$ & 4 & .56 & .31 & -.64 \\
\hline & 22 & 2.87 & 3 & .79 & .63 & 3.52 & 4 & .64 & .41 & -.65 \\
\hline \multirow{5}{*}{$\begin{array}{l}\text { Patrimonio mueble e } \\
\text { inmueble }\end{array}$} & 23 & 2.61 & 3 & .89 & .78 & 3.39 & 4 & .71 & .50 & -.78 \\
\hline & 24 & $2.24^{* *}$ & 2 & .90 & .80 & 3.12 & 3 & .83 & .69 & -.88 \\
\hline & 25 & 2.85 & 3 & .85 & .72 & 3.47 & 4 & .65 & .42 & -.62 \\
\hline & 26 & 2.69 & 3 & .91 & .83 & 3.26 & 3 & .80 & .65 & -.57 \\
\hline & 27 & 2.25 & 2 & .98 & .97 & $2.98 * *$ & 3 & .99 & .97 & -.73 \\
\hline
\end{tabular}

*Puntuación media más elevada; **puntuación media más baja

\section{Discusión}

Los resultados obtenidos en este estudio permiten constatar lo siguiente. En primer lugar, que cualquier propuesta formativa realizada en el ámbito de la escuela rural no puede realizarse al margen de la opinión ni de las necesidades de los propios implicados. Este hecho está en plena concordancia con lo expresado por la Comisión Europea (2005, 2007) a la hora de identificar y definir las competencias y procesos necesarios para una formación del profesorado de calidad, capaz de ejercer su función docente y social en cualquier contexto educativo.

Por otra parte, los resultados también permiten constatar que una propuesta formativa situada en el marco de la escuela rural, al margen de tener como referente competencias y saberes clave relacionados con el desempeño de la profesión docente, debe también contemplar el desempeño y la adquisición de las competencias y los saberes relacionados con la dimensión territorial. Este hecho se ajusta perfectamente a la recomendación de la Comisión Europea (2007) cuando sugiere que las propuestas formativas centradas en el dominio de competencias docentes deberán de adaptarse a las características del contexto, de los enseñantes y de las necesidades formativas de cada estado miembro.

A juzgar por los resultados obtenidos, si tan sólo se tienen en cuenta las puntuaciones medias obtenidas tanto para valorar aquellas competencias y saberes más relevantes como los que se tiene un dominio escaso, estas propuestas formativas, como mínimo, deberían de contemplar el desempeño de las competencias profesionales relacionadas con la dimensión territorial que se recogen en la tabla 5.

\section{Tabla 5. Competencias profesionales relacionadas con la dimensión territorial}

- Incluir en la planificación docente las características culturales de su territorio rural.

- Posibilitar acciones de desarrollo profesional para el profesorado del centro en relación con las necesidades formativas detectadas.

ÁMBITO METODOLÓGICO

- Incluir en la planificación docente las características culturales de su territorio rural.

- Incorporar el uso de TIC para el conocimiento de territorio rural próximo.

ÁMBITO RELACIÓN ESCUELA-COMUNIDAD

- Establecer procesos de participación escuela-familias-comunidad y viceversa.

- Incluir en los objetivos de la escuela el servicio al desarrollo cultural de su territorio rural.
} 
Y la adquisición estos saberes o contenidos específicos (tabla 6).

Tabla 6. Saberes relacionados con la dimensión territorial

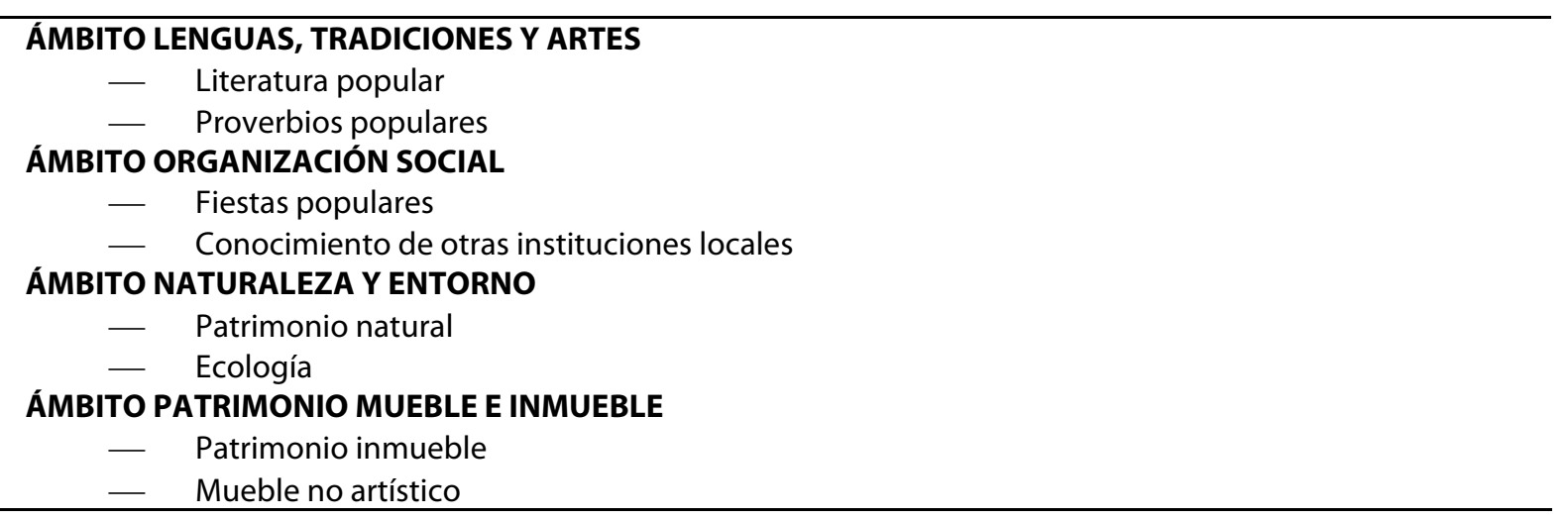

Obviamente, estas propuestas formativas también podrían construirse sobre la base del resto de competencias y saberes contemplados en los resultados. Sobre todo, si se tiene en cuenta que los resultados también han puesto de manifiesto la pertinencia de los marcos de referencia que se tuvieron en cuenta para la confección del catálogo de competencias (Comisión Europea, 2007; González y Wagenaar, 2010; Perrenaud, 2007).

En lo que respecta al tipo de formación que se podría planificar a partir de estos datos, es evidente que estas competencias y saberes pueden ser contemplados tanto en el marco de la formación inicial como permanente. En el caso de la formación inicial, quizá la vía más factible sería a través de una mención en escuela rural en los Grados de Maestro. Sin embargo, conviene recordar que, tanto en España como en Cataluña, no es necesario una especialidad en maestro rural para poder ejercer la profesión en estos contextos. Por tanto, a corto plazo, parece evidente que la vía natural a través de la cual se podría implantar esta formación específica sería la formación continuada, ya fuese a través de másteres universitarios (oficiales o no), cursos de postgrado o cursos de formación específica ofertada por las administraciones educativas.

Independientemente del modo empleado, es importante considerar que la realidad del territorio rural, así como de la práctica pedagógica de las escuelas multigrado no requieren de un maestro que sea un experto exclusivamente en escuela rural, sino de un maestro conocedor de la diversidad de centros escolares, y de las oportunidades que aportan las distintas maneras de organizar a los alumnos; un maestro que pueda ejercer tanto en una escuela ordinaria como en una escuela multigrado. Por tanto, el fin último de cualquier propuesta formativa en ambas tipologías de organización de alumnos (graduada y multigraduada) y de centro (rural y ordinaria) debe ser propiciar la mejora de la práctica pedagógica de ambas.

\section{Referencias}

Cano, E. (2011) Buenas prácticas en la evaluación de competencias. Barcelona, Laertes.

Comisión Europea (2004) Competencias clave para un aprendizaje a lo largo de la vida. Un marco de referencia europeo. Recuperado de http://www.educastur.princast.es/info/calidad/indicadores/doc/comision_europea. pdf_[Consulta: 24 de Octubre de 2013]. 
Convención para la salvaguardia del patrimonio cultural inmaterial (2003) [Documento en línea]. $\quad$ http://portal.unesco.org/es/ev.phpURL_ID=17716\&URL_DO=DO_TOPIC\&URL_SECTION=201.html_[Consulta: 25 de mayo de 2020].

European Commision (2005) Common European Principles for Teacher Competences and Qualifications. Education and Training. Brussels. [Documento en línea]. Recuperado de http://ec.europa.eu/education/policies/2010/doc/principles_en.pdf. [Consulta 25 de mayo de 2020]

European Commission (2007) Improving the Quality of Teacher Education. [Documento en línea]. Recuperado de https://eur-lex.europa.eu/legalcontent/EN/TXT/?uri=LEGISSUM\%3Ac11101 [Consulta: 25 de mayo de 2020]

European Comission (2012) Developing Key Competences at School in Europe: Challenges and Opportunities for Policy. Eurydice Report. Luxembourg: Publications Office of the European Union. [Documento en línea]. Recuperado de http://eacea.ec.europa.eu/education/eurydice/documents/thematic_reports/145EN .pdf [Consulta: 24 de octubre de 2013]

González, J. y Wagenaar, R. (2010) Tuning educational structures in Europe. Tuning Educational Structures in Europe. https://doi.org/10.1163/157007490X00133. [Consulta 25 de mayo de 2020].

McMillan, J. H. y Schumacher, S. (2011) Investigación educativa. Madrid, Pearson-Addison Wesley, $5^{\mathrm{a}}$ ed.

Oliver-Trobat, M., Forteza, D. y Urbina, S. (2015) «Análisis del perfil competencial del profesorado europeo». [Documento en línea] Profesorado: Revista de Currículum y Formación Del Profesorado, 19(2). Recuperado de http://www.ugr.es/local/recfpro/rev192COL3.pdf. [Consulta 25 de mayo de 2020]

Pereda, S; Berrocal, F. y López, M. (2002) «Gestión de recursos humanos y gestión del conocimiento». Dirección y organización, núm. 28, pp. 43-54.

Perrenaud, P. (2004) «La clave de los campos sociales: competencias del actor autónomo», en D. S. Rychen y L. H. Salganik (Eds.). Definir y seleccionar las competencias fundamentales para la vida. México DF, Fondo de Cultura Económica, pp. 216-261.

Perrenaud, P. (2007) Diez nuevas competencias para enseñar. Barcelona, Graó, $5^{\mathrm{a}}$ ed.

Spencer, L.M. y Spencer, S.M. (1993) Competence at work. Nueva York, John Wiley and sons, Inc.

UNESCO (2014) Indicadores de cultura para el desarrollo. Manual metodológico. [Documento en línea] Recuperado de https://es.unesco.org/creativity/sites/creativity/files/iucd_manual_metodologico_1. pdf_[Consulta: 25 de mayo de 2020].

Universidad de Deusto (2006) Normas y orientaciones para la elaboración de programas y guías de aprendizaje. Documento interno. 


\section{Orientacions per al disseny de plans de formació del professorat de les escoles ru- rals de Catalunya}

Resum: L'escola rural té una funció clau per arrelar la comunitat escolar amb el territori, i al mateix temps per visibilitzar i enfortir la seva presència a la població a la qual pertany. Per a això, el professorat d'aquestes escoles necessita d'una formació específica que li proporcioni els coneixements, les habilitats i les actituds que garanteixin l'acompliment d'aquesta funció. En aquest article es presenten unes orientacions per dissenyar aquesta formació específica fonamentades en els resultats obtinguts en el projecte FOPROMARErasmus plus (2017-1-ES01-KA201-038217). En concret es parteix de les dades obtingudes a partir del Qüestionari de Competències i Sabers del Professorat de l'Escola Rural, administrat als mestres i les mestres de les escoles rurals catalanes. Els resultats apunten que: 1 ) aquests plans de formació específica haurien de tenir present tant competències com sabers vinculats amb l'exercici de la dimensió territorial; 2) que les competències i sabers a seleccionar haurien de tenir en compte l'opinió del propi professorat.

Paraules clau: Formació de professorat, formació inicial, formació permanent, escola rural, dimensió territorial.

\section{Lignes directrices pour la conception de plans de formation pour les enseignants des écoles rurales de Catalogne}

Résumé: L'école rurale remplit une fonction clé pour ancrer la communauté scolaire dans le territoire et, en même temps, la rendre visible et renforcer sa présence au sein de la population à laquelle elle appartient. Pour ce faire, les enseignants de ces écoles ont besoin d'une formation spécifique qui leur fournisse les connaissances, les compétences et les attitudes nécessaires pour garantir l'accomplissement de cette fonction. Cet article présente des lignes directrices pour la conception de cette formation spécifique fondée sur les résultats obtenus dans le projet FOPROMAR-Erasmus plus (2017-1-ES01-KA201-038217). Plus précisément, il s'appuie sur les données extraites du questionnaire sur les compétences et les connaissances des enseignants de l'école rurale, réalisé auprès des enseignants des écoles rurales catalanes. Les résultats montrent que : 1) ces plans de formation spécifique devraient prendre en compte à la fois les compétences et les connaissances liées à la mise en œuvre de la dimension territoriale ; et 2) que les compétences et les connaissances à sélectionner devraient s'appuyer sur l'opinion du personnel enseignant.

Mots-clés: Formation des enseignants, formation initiale, formation continue, école rurale, dimension territoriale.

\section{Guidelines for designing teacher training plans for rural schools in Catalonia}

Abstract: Rural schools have a key function in involving the school community in their area and, at the same time, enhancing their visibility and strengthening their presence in the village they are located in. Teachers of rural schools need specific training to achieve all the skills and knowledge they need. This paper presents the main guidelines for designing this specific teacher training plan according the results obtained in the FOPROMAR-Erasmus plus project (2017-1-ES01-KA201-038217). We present the results obtained from the Questionnaire on the Teaching Skills of Rural School Teachers (CC-PER) answered by teachers who work in a rural schools in Catalonia ( $n=276$ ). The main results are: 1 ) All teacher training plans should include skills and knowledge related to the territorial dimension; 2) these skills and knowledge should be selected based on the rural school teachers' own opinions. The main conclusion is that the territorial dimension should be a key part of any specific teacher training plan.

Keywords: Teacher education, initial training, further training, rural school, territorial dimension. 